\title{
Correction to: A class of discrete dynamical systems with properties of both cellular automata and L-systems
}

\section{Roderick Edwards $^{1}$ (D) $\cdot$ Aude Maignan $^{2}$}

Published online: 19 September 2020

(C) Springer Nature B.V. 2020

\section{Correction to: Natural Computing (2020) 19:609-641 https://doi.org/10.1007/s1 1047-019-09739-5}

In the pdf version of the original publication, in the proof of Theorem 8 (second column of p. 632), the $T$ matrices appeared incorrectly. The correct values are given here.

$$
\begin{aligned}
T & =\left[\begin{array}{llllllll}
1 & 0 & 0 & 0 & 1 & 0 & 0 & 0 \\
1 & 0 & 0 & 0 & 1 & 0 & 0 & 0 \\
0 & 1 & 0 & 0 & 0 & 1 & 0 & 0 \\
0 & 1 & 0 & 0 & 0 & 1 & 0 & 0 \\
0 & 0 & 1 & 0 & 0 & 0 & 1 & 0 \\
0 & 0 & 1 & 0 & 0 & 0 & 1 & 0 \\
0 & 0 & 0 & 1 & 0 & 0 & 0 & 1 \\
0 & 0 & 0 & 1 & 0 & 0 & 0 & 1
\end{array}\right] \\
& =\left[\begin{array}{ll}
1 & 1
\end{array}\right] \otimes I_{4} \otimes\left[\begin{array}{l}
1 \\
1
\end{array}\right],
\end{aligned}
$$

$T=\left[\begin{array}{llll}0 & 0 & 0 & 0 \\ 0 & 0 & 1 & 0 \\ 0 & 1 & 0 & 1 \\ 0 & 1 & 0 & 1\end{array}\right]$

Publisher's Note Springer Nature remains neutral with regard to jurisdictional claims in published maps and institutional affiliations.

The original article can be found online at https:// doi.org/10.1007/s11047-019-09739-5.

Roderick Edwards

edwards@uvic.ca

Aude Maignan

aude.maignan@univ-grenoble-alpes.fr

1 Department of Mathematics and Statistics, University of Victoria, PO Box 1700 STN CSC, Victoria, BC V8W 2Y2, Canada

2 Univ. Grenoble Alpes, LJK, 51 rue des Mathematiques, B. P. 53, 38041 Grenoble Cedex 9, France 